facts regarding the influenza vaccination and rationale for HCW uptake. HCW who attended the intervention were offered a questionnaire at the educational session assessing their attitude towards the vaccination.

Result 157 HCW attended and 143 completed the questionnaire. In 2016/17 Influenza season 43.4\% $(n=62)$ indicated they received the influenza vaccination. Following the intervention, the intention to receive the vaccination in $2017 / 18$ increased to $76.2 \% \quad(n=109)$. A further $15.3 \%(n=22)$ indicated vaccination consideration. 50.3\% $(n=72)$ stated the brief intervention had influenced their decision. Prior to the educational intervention $39.2 \% \quad(n=56)$ agreed with mandatory Influenza vaccination for HCW. This increased to $49.7 \%$ $(\mathrm{n}=71)$ post intervention.

Discussion The brief educational intervention increased the numbers of HCW indicating they would get or would consider getting the annual Influenza vaccination. The intervention was noted as an influencing factor and could be a useful tool in increasing the vaccination rate amongst HCW in Ireland particularly if engagement with it was a mandatory requirement.

\section{HEALTH LITERACY IN JAPANESE WORKPLACE: ASSOCIATION WITH LIFESTYLE AND NCD'S}

${ }^{1}$ Fumiko Kitajima*, ${ }^{1}$ Hiroshi Fukuda, ${ }^{2}$ Yuka Sakamoto, ${ }^{2}$ Kayo Suwa, ${ }^{2}$ Akiko Fujiwara, ${ }^{2}$ Rie Morita. ${ }^{1}$ Department of General Medicine, School of Medicine, Juntendo University, Tokyo, Japan; ${ }^{2}$ Nikken Sekkei LTD, Tokyo, Japan

\subsection{6/oemed-2018-ICOHabstracts.477}

Introduction Health literacy (HL), which is defined as 'the ability to access, understand, and use information for health' (Nutbeam, 1998), has gained attention as a strategy to reduce health disparities, but not enough research has been conducted in Japanese workplace. This report shows to examine how HL among company employees are associated with lifestyle and NCD's.

Methods A cross-sectional study was conducted by giving selfadministered surveys to 2245 employees working at an architectural consultant company in 2014. Five questions from an communicative and critical HL scale for workers (Ishikawa, et al., 2008) were used, and simultaneously we asked Morimoto's lifestyle index including dietary, exercise, sleep, smoking and drinking habits, mental status of stress, and productivity. We also investigated the association between prevalence of obesity, hypertension, diabetes, dyslipidemia and HL from health screening data.

Results On the CCHL scales, scores for 'gathering information' were highest, with $60 \%$ of employees answering at least 'agree.' This was higher than 'selecting information', 'judging information', 'sharing information', and 'personal decisionmaking'. We found positive relation between HL and several lifestyles, such as concerning about diet balance, eating speed, late dinner, physical activity, quality of sleep. Self-awareness of stress was significantly low and the productivity was high in the high HL group. Prevalence of obesity, hypertension, dyslipidemia, diabetes are 21\%, 12\%, 35\%, 8\%, respectively. Prevalence of hypertension and diabetes are significantly low in the high HL group.

Conclusion Similar to previous study, we found the positive relation between employees HL and lifestyle or NCD's. This may be related to the high educational background of employees and the 15 years history of workplace health promotion activities carried out in this company. We will need to further analyse multi-facility and longitudinal analysis and conduct workplace health promotion activities according to employee's health literacy level.

\section{REBUILDING A CONCEPT FOR THE PERIODICAL HEALTH EXAMINATION IN JAPAN}

Yasushi Okubo*, Reiko Kuroda, Kenya Yamamoto. Division for Environment, Health and Safety, The University of Tokyo, Japan

\subsection{6/oemed-2018-ICOHabstracts.478}

Introduction The aims of the periodical health examination have been changed in accordance with social conditions and necessities in the ages. In past, the main aim was the prevention the occupational disease and work-related disease. In recent years, they are the prevention the metabolic syndrome and cardiac or cerebrovascular diseases. However, it is unclear the next step. Therefore, we investigated a concept of the periodical health examination for workers.

Methods We conducted reviews on the usability of the items of the periodical health examination and focus group discussions with the professional occupational health physicians. The first procedure was reviews of the items of the periodical health examination. The second procedure was the focus group discussion on the usability of the periodical health examination. Then we classified the issues into 5 groups. Each group made a concept of the health examination by focus group discussion.

Results Most of the items of the health examination were not effective to predict the onset the disease by the results of the literature reviews. However most of the occupational health physicians utilised each item in the health management activities. As the results of the focus group discussions, it was pointed out that the development the productivities and reduction the accidents at work should be adopted in the aims in the future, the fitness at work and the improvement the workers' health literacy should be used as the outcome, the prevention the cancers should be conducted as not the screening but the risk assessment.

Conclusion The health examination until now is the screening of the target diseases, but it should be developed into the evaluation the risk of the disease and working conditions, and the aims should be not only the workers' health but the development the productivities and the workers' health literacy.

\section{THERMAL STRESS AND CONSTRAIN IN A TUNISIAN STEEL INDUSTRY}

${ }^{1,2}$ Amira Omrane*, 1,2Lamia Bouzgarrou, ${ }^{1}$ Selma Kammoun, 1,2Awatef Kreim, ${ }^{1}$ Mohamed Adene Henchi, ${ }^{1,2}$ Taoufik Khalfallah. ${ }^{1}$ Research Unit, University of Monastir, Tunisia; ${ }^{2}$ Occupational Medicine Department, University Hospital, Taher Sfar, Mahdia, Tunisia

\subsection{6/oemed-2018-ICOHabstracts.479}

Introduction The hardship at work in the steel industry is linked to the important thermal constraints related to production processes. This study aims to identify the heat strain at work in the steel industry in Tunisia through the assessed metabolism by cardiovascular heart rate recording and to evaluate the thermal stress based on the various of measurable parameters. 